\title{
Designing Conservation Relations in Layered Synthetic Biomolecular Networks
}

\author{
Thomas P. Prescott and Antonis Papachristodoulou
}

\begin{abstract}
In Synthetic Biology, biomolecular networks are designed and constructed to perform specified tasks. Design strategies for these networks tend to center on tuning the parameters of mathematical models to achieve a specified behavior, and implementing these parameters experimentally. This design strategy often assumes a fixed network structure that defines the possible behaviors, which may be too restrictive for our purposes. This paper investigates the extent to which the state space of a synthetic network can also be designed and shaped by parametric tuning. We exploit timescale separation to implement new, nonlinear, tunable conservation relations that hold for all times beyond a fast transient. We demonstrate an application of this design strategy by flexibly constraining the possible behaviors of a gene regulatory network through the design of fast protein interactions.
\end{abstract}

Index Terms - Conservation relations, layers, singular perturbation, synthetic biology.

\section{INTRODUCTION}

$\mathbf{S}$ YNTHETIC Biology is a rapidly-developing field concerned with the de novo design or redesign of biomolecular circuits to specification. Fundamental current research centers on finding design principles for this task, which will speed the field's transition into a rigorous, standardized engineering discipline [1], [2].

Designing biomolecular networks requires extensive mathematical modelling, often identifying parameters that must be tuned to provide functionality that is robust to uncertainty and stochasticity. Tuned parameters may be implemented experimentally in synthetic networks by modulating specific biochemical properties [3]. There are many such 'dials' that can be tuned, at global level [4], [5], transcription level [6]-[8], or translation level [9], [10]. In particular, this article will consider systems tuned at a post-translational level [11], [12]. These 'dials' must be tuned subject to the uncertainty and stochasticity underlying the experimental realization of a mathematical model [13].

The parametric design process assumes that the network structure is chosen a priori, implying a set of given conservation relationships. A more ambitious design procedure would attempt to shape the conservation relationships themselves. The

Manuscript received February 13, 2015; revised June 02, 2015; accepted July 17, 2015. This work was supported by UK Engineering \& Physical Sciences Research Council grants EP/I031944/1 and EP/M002454/1. This paper was recommended by Associate Editor R. Sarpeshkar.

The authors are with the Department of Engineering Science, University of Oxford, Oxford OX1 3PJ, U.K. (e-mail: thomas.prescott@eng.ox.ac.uk).

Color versions of one or more of the figures in this paper are available online at http://ieeexplore.ieee.org.

Digital Object Identifier 10.1109/TBCAS.2015.2460376 question addressed in this paper is to determine when this can be done by designing the network's parameters. The ability to specify tunable nonlinear conservation relations will decouple the design of the allowed state values from the design of the system's dynamics.

The design strategy taken in this paper will impose timescale separation, which is characteristic of many evolved biomolecular networks [14] and has been widely applied to their analysis [15]-[21]. The principle of model reduction is that a system with a state in a high-dimensional space can be approximated by another system that evolves in a space of smaller dimension. A key feature of our previous work [21] is that this space is easily embedded in the original coordinates corresponding to biomolecular concentrations.

Various recent results apply timescale separation to the design of synthetic biocircuits. For example, to reduce the retroactivity [22] arising from composing multiple synthetic biomolecular gene transcription modules together, fast reactions were used in [23], [24] to enforce a desired signal direction. Conversely, a metabolic network can be steered to a given steady state by a slower genetic regulatory network (GRN) [25], or can be optimized for an arbitrary GRN [26].

Our approach is to constrain the state space of a synthetic biomolecular system with nonlinear conservation relations, implemented by designing a layer [27], [28] of additional fast reactions between the same species. In Section II, we outline the ODE model reduction technique used in Section III to identify the nonlinear conservation relations that can be shaped by the fast layer's design. Section IV applies this strategy to GRNs by designing protein interaction network (PIN) layers to implement particular nonlinear conservation relations. Finally, Section V discusses some practical aspects of the experimental implementation of layered design in Synthetic Biology, such as the effect of stochasticity on model reduction [29], [30] and the possible means of tuning post-translational biochemical parameters [11].

\section{PRELIMINARIES}

In this paper we consider dynamic ODE models of biomolecular reaction networks comprised of $n$ biochemical species $X_{i}$, for $i=1, \ldots, n$, and $m$ reactions $R_{j}$, for $j=1, \ldots, m[31]$. Define the vector $x(t)=\left[x_{1}(t), \ldots, x_{n}(t)\right]^{T}$ of scalar variables $x_{i}(t)$ that represent the concentration of $X_{i}$ at time $t \geq 0$. Define also the vector $v(x(t))=\left[v_{1}(x(t)), \ldots, v_{m}(x(t))\right]^{T}$ of reaction rates $v_{j}(x(t))$, which depend on the species concentrations $x$. The dynamics of $x$ are

$$
\dot{x}(t)=S v(x(t)), \quad x(0)=x_{0},
$$


for a specified initial condition vector $x_{0}$. Here, $S$ is the stoichiometry matrix of the network, mapping the reaction rates $v(x(t))$ to the rate of change of the concentrations $\dot{x}$.

A vector or matrix transpose is denoted with superscript.$^{T}$. For any given $n \times m$ matrix $M$ we write respectively

$$
\begin{aligned}
\operatorname{ker}(M) & =\left\{\mu \in \mathbb{R}^{m} \mid M \mu=0\right\}, \\
\operatorname{LNS}(M) & =\left\{\lambda \in \mathbb{R}^{n} \mid \lambda^{T} M=0\right\}, \\
\operatorname{Col}(M) & =\left\{\lambda \in \mathbb{R}^{n} \mid \exists \mu \in \mathbb{R}^{m}: \lambda=M \mu\right\}, \\
\operatorname{Row}(M) & =\left\{\mu \in \mathbb{R}^{m} \mid \exists \lambda \in \mathbb{R}^{n}: \mu^{T}=\lambda^{T} M\right\}
\end{aligned}
$$

for the kernel, left null space, column space, and row space.

Given the initial conditions $x(0)=x_{0}$ of (1), the state $x(t) \in$ $\mathbb{R}^{n}$ lies in a stoichiometric compatibility class [32], such that

$$
x(t)-x_{0} \in \operatorname{Col}(S)
$$

where $r=\operatorname{rank}(S)$ is the dimension of $\operatorname{Col}(S)$. Furthermore, each $x_{i}(t) \geq 0$ for all $t \geq 0$, as it represents a biochemical concentration.

Another interpretation of the stoichiometric compatibility class arises by considering the left null space $\operatorname{LNS}(S)$. For any $\lambda \in \operatorname{LNS}(S)$

$$
\lambda^{T} \dot{x}(t)=\left(\lambda^{T} S\right) v(x(t))=0
$$

independently of the flux vector $v(x(t))$. Hence for all $t$ the trajectory of $x(t)$ evolves such that $\dot{x}(t)$ is orthogonal to all $\lambda \in$ $\operatorname{LNS}(S)$. In particular, $\dot{x}(t)$ is orthogonal to each element of any set of $n-r$ independent $\lambda_{i}$ defining a basis for $\operatorname{LNS}(S)$. Each equation $\lambda_{i} \dot{x}(t)=0$ defines a linear conservation relation $\lambda_{i}^{T} x(t) \equiv \lambda_{i}^{T} x(0)=K_{i}$ for all $t \geq 0$, resulting in $n-r$ independent linear conservation relations.

The spaces $\operatorname{Col}(S)$ and $\operatorname{LNS}(S)$ defining the network's state space and conservation relations are independent of the reaction rates $v(x)$, and in particular of the model parameters. This paper will investigate to what extent the state space and conservation relations can be shaped by these parameters. Furthermore, the conservation relations $\lambda_{i}^{T} x(t) \equiv K_{i}$ are all linear. Can nonlinear constraints also be implemented? We will address these issues in Section III by applying previous results [21] on timescale-separated layers to identify the relationship between fast dynamics and conservation relations.

Consider a biomolecular reaction network with $m$ reactions proceeding at rates that separate in scale [15], [17], [21], [27]. For example, consider the model of the $P_{R M}$ operator region of $\lambda$ phage with $c I$ gene in [33]. The transcribed repressor is assumed to reversibly dimerize and bind to the promoters on a timescale on the order of seconds, compared to timescales of minutes for transcription and translation processes.

In general, the set $\mathcal{R}$ of reactions can be partitioned into disjoint subsets $\mathcal{R}^{s}$ and $\mathcal{R}^{f}$ of $m^{s}$ slow and $m^{f}$ fast reactions respectively. Then timescale separation can be parameterized by $0<\epsilon \ll 1$ such that (1) can be rewritten

$$
\begin{aligned}
\dot{x}(t) & =\left[\begin{array}{ll}
S^{s} & S^{f}
\end{array}\right]\left[\begin{array}{c}
\bar{v}^{s}(x) \\
\frac{\bar{v}^{f}(x)}{\epsilon}
\end{array}\right] \\
& =S^{s} \bar{v}^{s}(x(t))+\frac{1}{\epsilon} S^{f} \bar{v}^{f}(x(t))
\end{aligned}
$$

where, with no loss of generality, the indices of the columns of $S$ and elements of $v(x)$ are partitioned conformally with $\mathcal{R}^{s}$ and $\mathcal{R}^{f}$. The values in $\bar{v}^{f}$ and $\bar{v}^{s}$ are on the same scale, since the scale separation is captured by $\epsilon$.

Typically, model reduction by singular perturbation requires the classification of variables as fast or slow. In (2) this cannot be done; each species $X_{i}$ may take part in both slow and fast reactions. It was observed in [34] that combinations of apparently fast variables may exhibit slow dynamics. In this case, established approaches transform the state space into standard form to define timescale-separated variables [35]. However, in our previous work [21], [27], [28] we instead used layered decomposition to show the following result.

Definition 1: Given the timescale-separated system (2), the isolated fast and slow layers' states $x^{f}$ and $x^{s}$ have dynamics

$$
\begin{aligned}
\dot{x}^{f}(t) & =S^{f} \bar{v}^{f}\left(x_{0}+x^{f}(t)\right) \\
\dot{x}^{s}(t) & =S^{s} \bar{v}^{s}\left(x_{0}+x^{s}(t)\right)
\end{aligned}
$$

and initial conditions $x^{f}(0)=x^{s}(0)=0$.

Theorem 2: Consider any decomposition of $S^{f}=U^{f} C^{f}$ into full-rank matrices $U^{f} \in \mathbb{R}^{n \times r^{f}}$ and $C^{f} \in \mathbb{R}^{r^{f} \times m^{f}}$, where $r^{f}=\operatorname{rank}\left(S^{f}\right)$. For a state $\tilde{x}$ taking values $\tilde{x}(t) \in \mathbb{R}^{n}$ define the dynamics

$$
\dot{\tilde{x}}=\left[I-M^{f}(\tilde{x})\right] S^{s} \bar{v}^{s}(\tilde{x})
$$

for the state-dependent matrix

$$
M^{f}(\tilde{x})=U^{f}\left(C^{f} \partial \bar{v}^{f}(\tilde{x}) U^{f}\right)^{-1} C^{f} \partial \bar{v}^{f}(\tilde{x})
$$

where $\partial \bar{v}^{f}(\tilde{x})$ denotes the Jacobian of $\bar{v}^{f}$ evaluated at $\tilde{x}$. Assume that the initial condition of (5) is $\tilde{x}(0)=x_{0}+\phi\left(x_{0}\right)$, where $\phi\left(x_{0}\right)=\lim _{t \rightarrow \infty} x^{f}(t)$ is the steady state of the isolated fast layer's dynamics (3).

As $\epsilon \rightarrow 0$ the trajectory $\tilde{x}(t)$ satisfying (5) arbitrarily closely approximates (in $\mathcal{L}_{2}$ ) the trajectory $x$ satisfying (1).

Proof: This result follows from Tikhonov's Theorem [36] applied to (2) [21]. The key to the proof is that $-M^{f}(\tilde{x})=$ $\mathrm{d} \tilde{x}^{f}\left(\tilde{x}^{s}\right) / \mathrm{d} \tilde{x}^{s}$ maps the nominal evolution $S^{s} v^{s}(\tilde{x})$ of the isolated slow layer to the response of the fast layer, providing a 'correction' perturbation as the fast reactions equilibrate.

The timescale-separated ODE (2) represents the feedback interconnection of a fast and slow layer. The singular perturbation of this system results in (5), where the fast dynamic layer has been approximated as a static layer. The dynamics in (5) are a projected version of the slow layer's isolated dynamics (4), where the pre-multiplying matrix $P^{f}(\tilde{x})=I-M^{f}(\tilde{x})$ is defined by the fast layer's structure $S^{f}$ and reactions $\bar{v}^{f}$.

\section{FAST LAYERS AND CONSERVATION RELATIONS}

\section{A. Model Reduction}

Recall that $n-r$ independent conservation relations in the general ODE model (1) are defined by the elements $\lambda_{i} \in \operatorname{LNS}(S)$ of any basis for the left null space of $S$. For the layered ODE (2), these conservation relations are defined by the left null space of $S=\left[S^{s} S^{f}\right]$. In other words, the state 


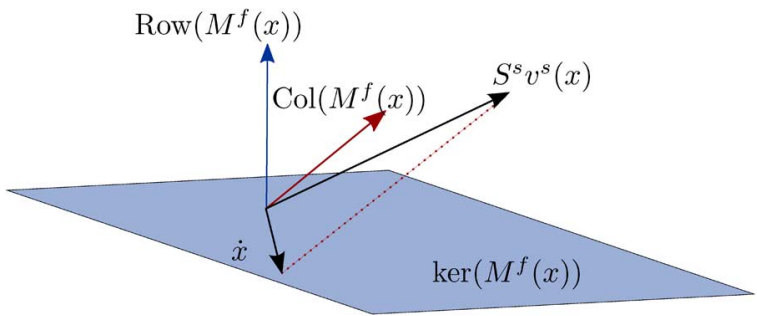

Fig. 1. Schematic diagram illustrating the effect of the fast layer on the slow layer, implied by (5), dropping tilde notation. The isolated slow dynamics $S^{s} v^{s}(x)$ are projected into $\operatorname{ker}\left(M^{f}(x)\right)$ along $\operatorname{Col}\left(M^{f}(x)\right)$, as shown by the parallel red lines. This projection is generally non-orthogonal, as $\operatorname{Row}\left(M^{f}(x)\right) \neq \operatorname{Col}\left(M^{f}(x)\right)$.

space is defined by the combined stoichiometric matrices of the slow and fast layers.

However, consider instead the approximate model (5), dropping the ? for simplicity. The matrix $M^{f}(x)$ in (6) is clearly a projection, and thus so too is the perturbation $P^{f}(x)$ in (5). Fig. 1 depicts the effect of $P^{f}(x)$ on the isolated slow dynamics at a given state value $x$. It is easy to show that $\operatorname{Col}\left(P^{f}(x)\right)=\operatorname{ker}\left(M^{f}(x)\right)$, implying that the derivative $\dot{x}(t)$ in (5) always takes values in the kernel of $M^{f}(x)$. In other words, the linear space $\operatorname{ker}\left(M^{f}(x)\right)$ lies tangent to the nonlinear slow manifold defined by $S^{f} v^{f}(x)=0$. Furthermore, the associated fibres [37], locally to the slow manifold, are in the space $\operatorname{Col}\left(M^{f}(x)\right)$. The equality $\operatorname{Col}\left(M^{f}(x)\right)=\operatorname{ker}\left(P^{f}(x)\right)$ implies that if $S^{s} v^{s}(x)$ is in the column space of $M^{f}(x)$, then $P^{f}(x) S^{s} v^{s}(x)=0$, and the approximated system is thus at steady state, even when the isolated slow dynamics are non-zero.

As defined in Section II, conservation relations in (5) are defined by vectors $\lambda \in \mathbb{R}^{n}$ satisfying $\lambda^{T} \dot{x}=0$ for any slow flux vector $v^{s}(x)$. The $\lambda$ discussed in Section II are constant, and therefore apply for all state values. However, the following result is local, in the sense that an arbitrary state value $x$ is fixed. We then seek the left null space $\Lambda(x)=\operatorname{LNS}\left(P^{f}(x) S^{s}\right)$, which is clearly state dependent. The following result is closely related to the identification of the equilibrium or slow manifold [18], [37], [38].

Theorem 3: Fix a state value $x \in \mathbb{R}^{n}$. The left null space $\Lambda(x)=\left\{\lambda \in \mathbb{R}^{n} \mid \lambda^{T} P^{f}(x) S^{s}=0\right\}$ defining the vector space orthogonal to the approximated model (5) is given by

$$
\begin{aligned}
\Lambda(x) & =\operatorname{Row}\left(M^{f}(x)\right) \oplus\left(\operatorname{LNS}\left(S^{s}\right) \cap \operatorname{LNS}\left(S^{f}\right)\right) \\
& =\operatorname{Row}\left(C^{f} \partial \bar{v}^{f}(x)\right) \oplus\left(\operatorname{LNS}\left(S^{s}\right) \cap \operatorname{LNS}\left(S^{f}\right)\right)
\end{aligned}
$$

where, for each layer $l=f, s$, the stoichiometric matrices can be decomposed into $S^{l}=U^{l} C^{l}$ for full-rank matrices $U^{l} \in$ $\mathbb{R}^{n \times r^{l}}$ and $C^{l} \in \mathbb{R}^{r^{l} \times m^{l}}$, with $r^{l}=\operatorname{rank}\left(S^{l}\right)$.

Proof: The transpose of any projection is also a projection. Hence, any $\lambda \in \mathbb{R}^{n}$ can be written as the unique sum $\lambda=$ $\lambda_{M}+\lambda_{P}$ of $\lambda_{M} \in \operatorname{Col}\left(M^{f}(x)^{T}\right)$ and $\lambda_{P} \in \operatorname{Col}\left(P^{f}(x)^{T}\right)$. Clearly $\operatorname{Col}\left(M^{f}(x)^{T}\right)=\operatorname{Row}\left(M^{f}(x)\right)$, and

$$
\operatorname{Col}\left(P^{f}(x)^{T}\right)=\operatorname{ker}\left(M^{f}(x)^{T}\right)=\operatorname{LNS}\left(M^{f}(x)\right)=\operatorname{LNS}\left(S^{f}\right) .
$$

We proceed to show (7) by showing both inclusions.
Consider any $\lambda \in \Lambda(x) \subseteq \mathbb{R}^{n}$, uniquely decomposed into $\lambda=\lambda_{M}+\lambda_{P}$ with $\lambda_{M} \in \operatorname{Row}\left(M^{f}(x)\right)$ and $\lambda_{P} \in \operatorname{LNS}\left(S^{f}\right)$. To show that $\Lambda(x)$ is a subset of the right-hand side of (7) it remains only to show also that $\lambda_{P} \in \operatorname{LNS}\left(S^{s}\right)$. Since $\lambda_{M}^{T} P^{f}(x)=0$ and $\lambda_{P}^{T} P^{f}(x)=\lambda_{P}^{T}$, the equation

$$
0=\left(\lambda_{M}+\lambda_{P}\right)^{T} P^{f}(x) S^{s}=\lambda_{P}^{T} P^{f}(x) S^{s}=\lambda_{P}^{T} S^{s}
$$

implies that $\lambda_{P} \in \operatorname{LNS}\left(S^{s}\right)$.

To show the reverse inclusion, consider any $\lambda \in \operatorname{Row}\left(M^{f}\right) \oplus$ $\left(\operatorname{LNS}\left(S^{s}\right) \cap \operatorname{LNS}\left(S^{f}\right)\right)$, decomposed into $\lambda=\lambda_{M}+\lambda_{P}$. Clearly $\lambda_{M}^{T} P^{f}(x)=0$, from which it follows that $\lambda_{M} \in$ $\Lambda(x)$. Finally, $\lambda_{P} \in \operatorname{LNS}\left(S^{f}\right)=\operatorname{Col}\left(P^{f}(x)^{T}\right)$ implies that $\lambda_{P}^{T} P^{f}(x)=\lambda_{P}^{T}$. Since also $\lambda_{P} \in \operatorname{LNS}\left(S^{s}\right)$, it follows that $\lambda_{P}^{T} P^{f}(x) S^{s}=\lambda_{P}^{T} S^{s}=0$ and hence that $\lambda_{P} \in \Lambda(x)$.

This result means that each independent conservation relation for (5) is one of two types. First, any element $\lambda_{M} \in \operatorname{Row}\left(M^{f}(x)\right)$ satisfies $\lambda_{M}^{T} \dot{x}=0$. Thus

$$
C^{f} \partial \bar{v}^{f}(x(t)) \dot{x}(t)=0
$$

and hence $C^{f} \bar{v}^{f}(x(t)) \equiv K$ for a constant vector $K \in \mathbb{R}^{r^{f}}$, for all $t \geq 0$. The initial conditions of (5) given in Theorem 2 imply that $K=0$, assuming that the isolated fast layer (3) is stable. Hence $\operatorname{Row}\left(M^{f}\right)$ generates $r^{f}$ conservation relations

$$
C^{f} \bar{v}^{f}(x(t)) \equiv 0
$$

for all $t \geq 0$. These conservation relations are nonlinear if $\bar{v}^{f}$ is nonlinear, and depend on the parameters (apart from $\epsilon$ ) used to define the fast reactions $v^{f}$. However, they only apply after a fast transient, during which the exact trajectory of (2) quickly approaches the space defined by (8).

The second class of conservation relations is given by $\lambda_{P} \in$ $\operatorname{LNS}\left(S^{s}\right) \cap \operatorname{LNS}\left(S^{f}\right)$. It is the space of conservation relations that apply on both isolated timescales (3) and (4). These conservation relations must be linear, and are independent of the parameters of the fast network, being instead characterized by the stoichiometric structure of both layers.

To illustrate how Theorem 3 corresponds to a layered network's conservation relations, consider the toy system

$$
X_{1} \underset{\beta_{1}}{\stackrel{\alpha_{1}}{\rightleftharpoons}} X_{2} \underset{\frac{\beta_{2}}{\epsilon}}{\stackrel{\frac{\alpha_{2}}{\epsilon}}{\epsilon}} X_{3}
$$

where $0<\epsilon \ll 1$ parameterises the timescale separation. The exact ODE for this system has stoichiometric matrix $S$ with $r$ $=\operatorname{rank}(S)=2$ and conservation $x_{1}+x_{2}+x_{3} \equiv K$ so that the state values lie in a plane embedded in $\mathbb{R}^{3}$. However, the dynamics of this system can be approximated as (5) using Theorem 2. We calculate

$$
M^{f}(x)=\frac{1}{\alpha_{2}+\beta_{2}}\left[\begin{array}{ccc}
0 & 0 & 0 \\
0 & -\alpha_{2} & \beta_{2} \\
0 & \alpha_{2} & -\beta_{2}
\end{array}\right]
$$

according to the formula in (6) to give

$$
\dot{x}=\left[\begin{array}{ccc}
1 & 0 & 0 \\
0 & \gamma & \gamma \\
0 & 1-\gamma & 1-\gamma
\end{array}\right]\left[\begin{array}{c}
-1 \\
1 \\
0
\end{array}\right]\left(\alpha_{1} x_{1}-\beta_{1} x_{2}\right)
$$


where $\gamma=\beta_{2} /\left(\alpha_{2}+\beta_{2}\right)$ and $\sim$ notation is dropped. Note that $M^{f}(x)$ is independent of $x$ because the dynamics are linear.

The vectors $\lambda \in \operatorname{Row}\left(M^{f}\right)=\operatorname{Span}\left(\left[0 \alpha_{2}-\beta_{2}\right]^{T}\right)$ clearly satisfy $\lambda^{T} \dot{x}=0$. Furthermore, both two-dimensional left nullspaces of $S^{s}$ and $S^{f}$ intersect to give $\operatorname{Span}\left(\left[\begin{array}{ll}1 & 1\end{array}\right]^{T}\right)$. Thus two independent conservation relations of this system are defined by $\lambda_{1}=\left[\begin{array}{lll}0 & \alpha_{2} & -\beta_{2}\end{array}\right]^{T}$ and $\lambda_{2}=\left[\begin{array}{lll}1 & 1 & 1\end{array}\right]^{T}$. Clearly, $\lambda_{1} \in$ Row $\left(M^{f}(x)\right)$ depends on the fast layer's parameters $\alpha_{2}$ and $\beta_{2}$, and can be written in the form (8) as $\alpha_{2} x_{2}-\beta_{2} x_{3} \equiv 0$. However, $\lambda_{2} \in \operatorname{Col}\left(S^{s}\right) \cap \operatorname{Col}\left(S^{f}\right)$ is independent of $\alpha_{2}$ and $\beta_{2}$.

\section{B. Designing Conservation Relations}

The results in Section III-A suggest that timescale separation can be exploited to design approximate constraints on a system's state values for times beyond a fast transient.

Consider a given network that can be decomposed into fast and slow layers based on reaction speeds. The conservation relations of its exact model (2) correspond to the left null space $\operatorname{LNS}(S)$ of $S=\left[S^{s} S^{f}\right]$. However, we have shown that, beyond a fast transient, the approximated state satisfies the conservation relations (7). If $r^{f}=\operatorname{rank}\left(S^{f}\right)$, then $r^{f}$ of these conservation relations are in general tunable, since they depend on fast reactions' parameters, and nonlinear, since they are defined by a fast flux vector $\bar{v}^{f}(x)$ that may be nonlinear in $x$. Importantly, the conservation relations (7) are independent of $v^{s}(x)$, so that the conservation relations and slow dynamics can be designed independently.

We can exploit this principle for Synthetic Biology as follows. Suppose that all reactions of a given network occur on the same timescale, with a fixed state space of dimension $r^{s}$ (embedded in $\mathbb{R}^{n}$ ) defined by $n-r^{s}$ linear conservation relations. Now suppose that we wish to redesign the behavior of this network to satisfy new conservation relations. The approach that will be taken is to construct a fast layer (that is, implement a number of fast reactions) that enforces the specified constraints on the slow timescale, guaranteed to hold for any design of its slow dynamics.

The first part of $\Lambda(x)$ in (7) implies that there are $r^{f}$ nonlinear, tunable conservation relations. The second component means that, in addition, the fast layer's stoichiometric matrix can be designed to remove at most $r^{f}$ of the original $n-r^{s}$ linear conservation relations. Assuming that $r^{s}=n$, so that the isolated slow layer has no conservation relations, then the only conservation relations resulting from the fast layer will be the $r^{f}$ nonlinear algebraic equations $C^{f} \bar{v}^{f}(x) \equiv 0$.

\section{Constraining Genetic Regulation}

Consider a gene regulatory network (GRN) of $n$ genes, each producing a transcription factor (TF) $X_{i}$ for $i=1, \ldots, n$. Let $x_{i}(t)$ represent the concentration of $X_{i}$ at time $t$. This network can be modelled by the $n$ coupled ODEs

$$
\dot{x}_{i}(t)=\alpha_{i}-\delta_{i} x_{i}(t)+R_{i}(x(t))
$$

for $x=\left(x_{1}, \ldots, x_{n}\right)^{T}$. The terms $R_{i}(x)$ define how the TFs regulate the expression of $X_{i}$. In the notation of (1), the stoichiometric matrix is the identity $S=I$; there are no conservation relations, since $r^{s}=n$.
Typically, the terms $R_{i}(x)$ are the primary target of the design effort for synthetic GRNs. For example, if the expression of $X_{2}$ is promoted by $X_{1}$, we may model this by

$$
R_{2}(x)=\frac{\beta_{2}\left(\frac{x_{1}}{K_{2}}\right)^{n}}{1+\left(\frac{x_{1}}{K_{2}}\right)^{n}}
$$

where the parameters, corresponding to experimentally implemented 'dials', may be tuned to produce specified behavior [3]. In this paper we propose a complementary approach to GRN design that overlays a fast layer designed to constrain the possible values of the state $x$, independently of $\alpha_{i}, \delta_{i}$ and $R_{i}(x)$. The fast layer will take the form of a protein interaction network (PIN) involving direct interactions between TFs; as is common, the PIN reactions are assumed to be much faster than GRN processes [23]. Given the fast layer and resulting conservation relations, the GRN (9) can be designed separately. This layered structure reflects design principles of evolved networks that combine genetic regulation with protein interaction [39].

\section{A. One Nonlinear Conservation Relation}

For example, consider the nonlinear constraint $x_{3}=\gamma x_{1} x_{2}$ on the state-space of the GRN (9), for $n \geq 3$. We make no assumptions on the slow parameters $\alpha_{i}$ and $\delta_{i}$ or the coupling terms $R_{i}(x)$. The required conservation relation is implemented by introducing the following direct interactions between the TFs $X_{i}$ under arbitrary genetic regulation.

1) Implementation One: Consider the single fast reversible reaction

$$
X_{1}+X_{2} \underset{\frac{\beta}{\epsilon}}{\stackrel{\frac{\alpha}{\epsilon}}{\epsilon}} X_{3}
$$

for the small parameter $0<\epsilon \ll 1$. For example, each reaction direction may be implemented by introducing two enzymes, whose concentrations can tune the parameters $\alpha$ and $\beta$ respectively. When this fast layer, of dimension $r^{f}=1$, is integrated with the slower GRN layer the resulting dynamics can be modelled by (5). Then $M^{f}(x)$ is given by (6) as

$$
M(x)=\frac{1}{\pi(x)}\left[\begin{array}{c}
1 \\
1 \\
-1 \\
0 \\
\vdots \\
0
\end{array}\right]\left[\begin{array}{llllll}
\alpha x_{2} & \alpha x_{1} & -\beta & 0 & \ldots & 0
\end{array}\right]
$$

with $\pi(x)=\alpha\left(x_{1}+x_{2}\right)+\beta$. For any fixed state vector $x$, the set $\Lambda(x)$ defining the conservation relations in the system can be deduced from (7) as the $r^{f}=1$-dimensional space

$$
\begin{aligned}
\Lambda(x) & =\operatorname{Row}\left(M^{f}(x)\right) \\
& =\operatorname{Span}\left(\left[\begin{array}{lllllll}
\alpha x_{2} & \alpha x_{1} & -\beta & 0 & \ldots & 0
\end{array}\right]^{T}\right)
\end{aligned}
$$

which is clearly both parameter- and state-dependent. Note that are no conservation relations arising from the space $\operatorname{LNS}\left(S^{f}\right) \cap$ $\operatorname{LNS}\left(S^{s}\right)$, since $\operatorname{LNS}\left(S^{s}\right)=\{0\}$.

Equation (8) implies that the single conservation relation defined by $\operatorname{Row}\left(M^{f}\right)$ is given by the expression 


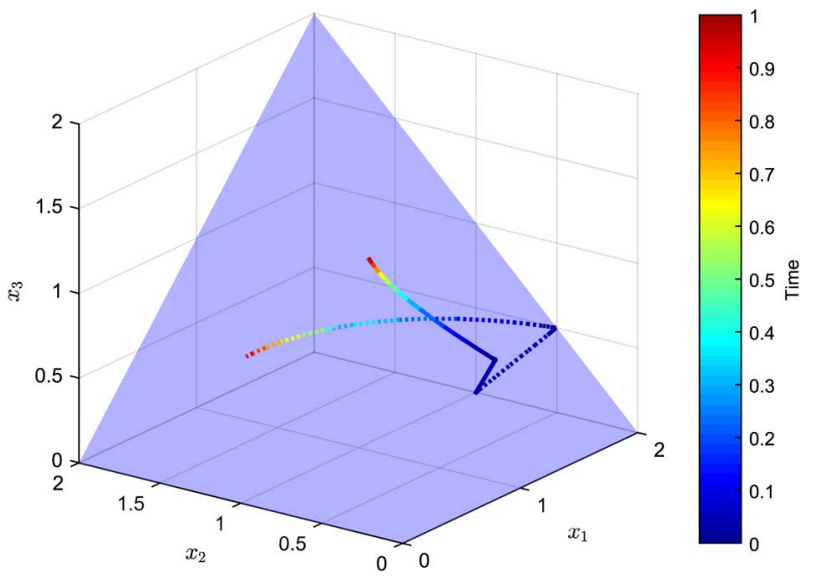

Fig. 2. Two implementations of constraint $x_{3}=\gamma x_{1} x_{2}$ [blue surface] with slow layer (9) and fast layers (10) [solid line] and (11) [dotted line]. The slow layer's parameters are $n=3,\left(\alpha_{1}, \alpha_{2}, \alpha_{3}\right)=(1,2,3),\left(\beta_{1}, \beta_{2}, \beta_{3}\right)=$ $(2,3,1)$, and $R_{i}=0$; both fast layers' parameters are $\alpha=1, \beta=2$, and $\epsilon=0.001$, to give $\gamma=1 / 2$. Initial conditions are $x_{0}=(2,1,0)^{T}$.

$$
x_{3}=\frac{\alpha}{\beta} x_{1} x_{2}
$$

Hence, tuning the fast reaction rates in the PIN (10) such that $\alpha$ $=\beta \gamma$ ensures that the required constraint $x_{3}=\gamma x_{1} x_{2}$ holds at times beyond a fast transient for any design of the GRN (9).

2) Implementation Two: Alternatively, the same conservation relation could be implemented by the catalytic reaction

$$
\begin{gathered}
X_{1} \\
\downarrow X_{2} \underset{\frac{\alpha}{\epsilon}}{\stackrel{\frac{\alpha}{\epsilon}}{\rightleftharpoons}} X_{3}
\end{gathered}
$$

where $X_{1}$ is neither consumed nor produced, but does influence the forward reaction rate. Assuming that the forward reaction rate is linear in $x_{1}$ implies the same form for $\bar{v}^{f}=\alpha x_{1} x_{2}-\beta x_{3}$, and hence the same conservation relation.

Although the resulting conservation relations are equal, there is an important difference in behavior between the implementations (10) and (11), as depicted in Fig. 2. Both trajectories lie on the surface $x_{3}=\gamma x_{1} x_{2}$, for $t$ beyond a fast transient. However, the trajectories on this surface, given identical slow layers, are very different.

Consider each of the two implementations' column spaces $\operatorname{Col}\left(M^{f}\right)=\operatorname{Col}\left(S^{f}\right)$. For (10) this space is spanned by $(-1,-1,1,0, \ldots, 0)^{T}$, while for (11) it is spanned by $(0,-1,1,0, \ldots, 0)^{T}$. Recall from Fig. 1 that these spaces define how the slow dynamics project onto $\operatorname{Row}\left(M^{f}\right)$. Hence using (10) implies that $\dot{x}_{1}, \dot{x}_{2}$ and $\dot{x}_{3}$ are projected by $P^{f}$, while using (11) implies that $P^{f}$ will not affect $\dot{x}_{1}$, so that $X_{1}$ remains under genetic control only.

Similarly, the steady state of the isolated fast layer (3) changes depending on its stoichiometric matrix. Hence, the choice of implementation also determines the approximate 'initial condition' given in Theorem 2, corresponding to the state value after the fast transient. The point at which the (exact) trajectory joins the specified state space therefore depends on the fast layer's implementation. For example, Fig. 2 shows that for (11) the value of $x_{1}(t)$ remains approximately constant at $x_{1}(0)=2$ while $x_{2}(t)$ and $x_{3}(t)$ quickly equilibrate over small values of $t \ll 1$.

\section{B. Combining Conservation Relations}

As another example of a possible nonlinear state space constraint, consider the parabolic relationship $x_{3}=\gamma x_{1}^{2}$. Again, we propose two possible fast layer implementations.

1) Implementation One: Consider first the fast reaction

$$
2 X_{1} \underset{\frac{\underline{\beta}}{\epsilon}}{\stackrel{\frac{\alpha}{\epsilon}}{\rightleftharpoons}} X_{3}
$$

where again $0<\epsilon \ll 1$. It is easy to show that $x_{3}=(\alpha / \beta) x_{1}^{2}$, implied by (8), will hold for times beyond the fast transient.

Fig. 3 displays the trajectory resulting from this implementation. We plot the constraint $x_{3}=\gamma x_{1}^{2}$ in $\mathbb{R}^{3}$ and $\mathbb{R}^{2}$, together with a simulated trajectory of the layered system (2), where for simplicity the TFs are decoupled genetically through putting $R_{i}=0$. Beyond the fast transient, the state values are clearly constrained by the designed conservation relation.

2) Implementation Two: Alternatively, the fast PIN could be comprised of two reactions

$$
\begin{array}{r}
X_{1}+X_{2} \underset{\frac{\underline{\beta}}{\epsilon}}{\stackrel{\frac{\alpha}{\epsilon}}{\rightleftharpoons}} X_{3}, \\
X_{1} \underset{\frac{\frac{\delta}{\epsilon}}{\epsilon}}{\stackrel{\frac{\kappa}{\epsilon}}{\rightleftharpoons}} X_{2} .
\end{array}
$$

This layer is now of dimension $r^{f}=2$, implying that two conservation relations hold. The relationship $\beta x_{3}=\alpha x_{1} x_{2}$ holds as a result of the first reaction, and the second implies that $\kappa x_{1}=\delta x_{2}$. Thus designing parameters such that $\gamma=\alpha \kappa / \beta \delta$ will ensure that $x_{3}=\gamma x_{1}^{2}$.

Similarly to Fig. 3, Fig. 4 depicts this implementation. Each blue surface in Fig. 4(a) corresponds to one of the two independent conservation relations, while the curve in Fig. 4(b) is a projection of their intersection into $x_{1} / x_{3}$-space. In both plots, we show a simulation of a representative GRN integrated with the PIN (13). Clearly, past a fast transient, the state values lie in the intersection of the $r^{f}=2$ independent conservation relations, which can be implemented separately.

This example demonstrates that fast reactions can be combined to achieve specified conservation relations. However, the dimension $r^{f}$ of the fast layer will tend to increase when combining multiple reactions, imposing additional conservation relations and leading to further constraints. This may result in some redundancy in the tuning strategy: in this example, halving $\kappa$ and doubling $\beta$ gives the same value for $\gamma$, and hence the same specified behavior in $\mathbb{R}^{2}$. However, this choice results in significantly different behavior in $\mathbb{R}^{3}$, as the new parameters reshape both surfaces in Fig. 4(a).

\section{Fast Layers and Signal Propagation}

A potential application for this design approach is to enforce a signal direction in the underlying GRN. Consider an example of $n=4$ genes where the TF products $X_{1}$ and $X_{2}$ co-regulate one another, and likewise for $X_{3}$ and $X_{4}$, but where there is no 


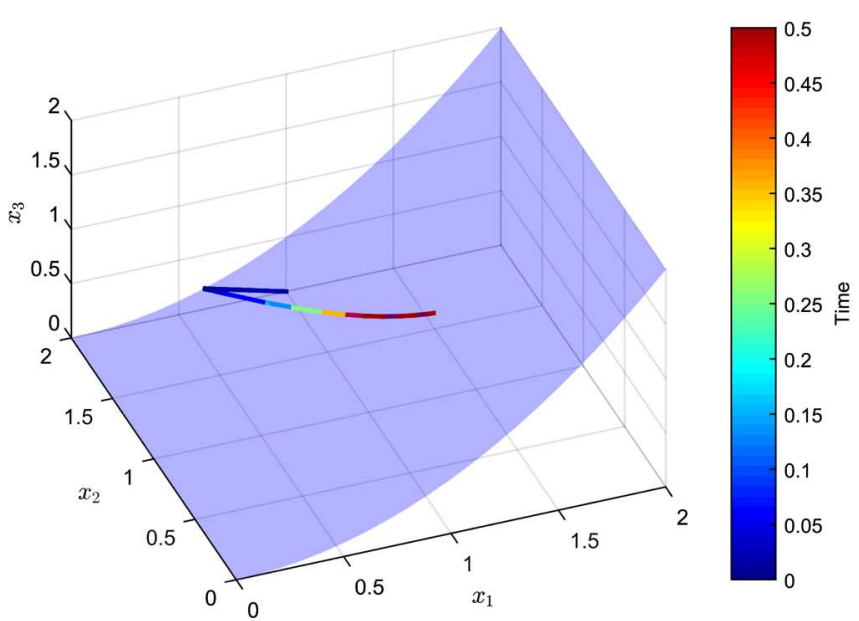

(a)

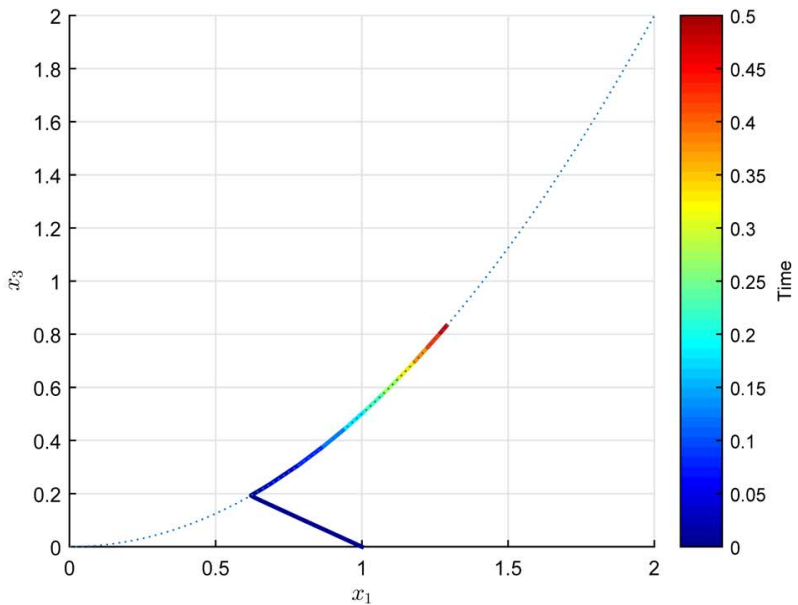

(b)

Fig. 3. Implementation of constraint $x_{3}=\gamma x_{1}^{2}$ with slow layer (9) and fast layer (12) of dimension $r^{f}=1$. All parameter values are as in Fig. 2, with initial conditions $x_{0}=(1,2,0)^{T}$. (a) Projection of coordinates $\left(x_{1}, x_{2}, x_{3}\right) \in \mathbb{R}^{3}$. (b) Projection of coordinates $\left(x_{1}, x_{3}\right) \in \mathbb{R}^{2}$.

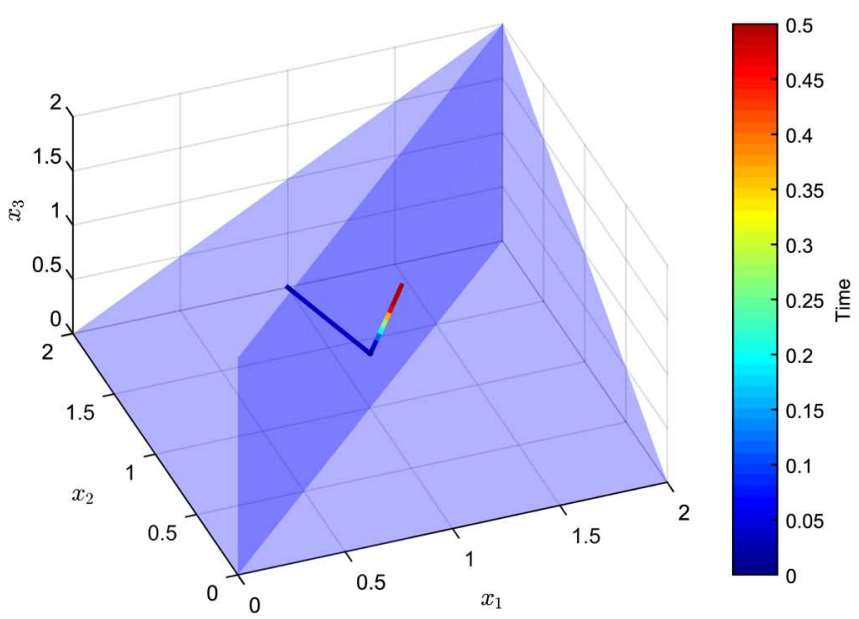

(b)

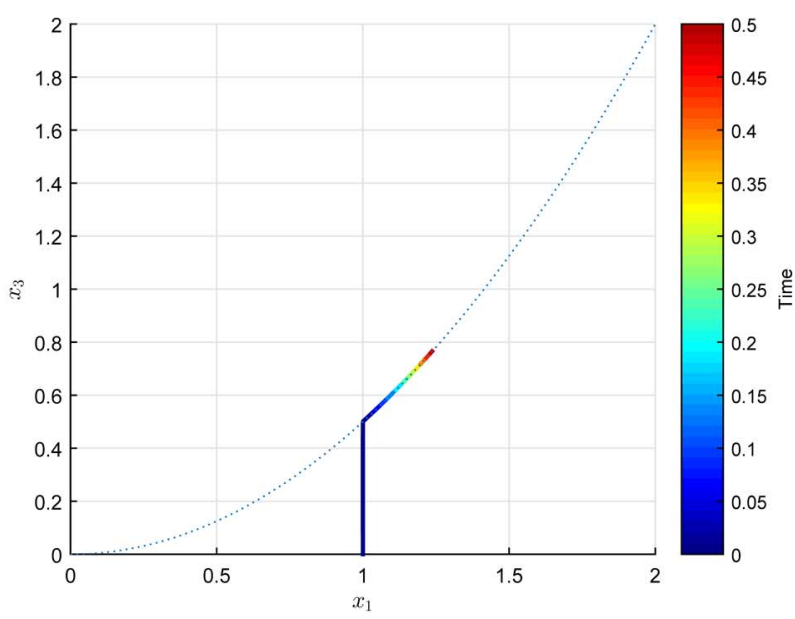

(b)

Fig. 4. Implementation of constraint $x_{3}=\gamma x_{1}^{2}$ with slow layer (9) and fast layer (13) of dimension $r^{f}=2$. The systems have the same parameter values and initial conditions as in Fig. 3, and additionally $\kappa=\delta=2$ so that $\gamma=1 / 2$. (a) Projection of coordinates $\left(x_{1}, x_{2}, x_{3}\right) \in \mathbb{R}^{3}$. (b) Projection of coordinates $\left(x_{1}, x_{3}\right) \in \mathbb{R}^{2}$.

direct cross-regulation between these two TF pairs. A typical approach to connect these two modules might be to redesign the promoters of genes 3 and 4 so that $R_{3}(x)$ or $R_{4}(x)$ also depend on $x_{1}$ or $x_{2}$, thus putting the $X_{3} / X_{4}$ module downstream of $X_{1} / X_{2}$. However, the behavior of the upstream module is likely to change as a result of the load from the downstream module [22], [40].

Our design strategy is to instead move the coupling design into a fast PIN layer. Note that this is not the same as using $X_{1}$ or $X_{2}$ as an input into the genetic regulation of $X_{3}$ or $X_{4}$. Rather, it couples the modules by enforcing the concentrations to satisfy a specified conservation relation. Nevertheless, as discussed in Section IV-A, the chosen implementation will affect signal directionality.

For example, the modules can be coupled such that $x_{2}=x_{3}$. There are at least three possible implementions of this relation by a fast PIN layer. First is the reversible conversion

$$
X_{2} \underset{\frac{1}{\epsilon}}{\stackrel{\frac{1}{\epsilon}}{\rightleftharpoons}} X_{3}
$$

where the fast forward and reverse rates are tuned to be equal. An alternative is to consider an additional protein $Y_{1}$ such that

$$
\begin{aligned}
& \emptyset \stackrel{\frac{K}{\epsilon}}{\longrightarrow} Y_{1} \stackrel{\frac{\delta}{\epsilon}}{\longrightarrow} \emptyset \\
& Y_{1} \underset{\frac{\alpha}{\epsilon}}{\stackrel{\frac{\beta}{\epsilon}}{\epsilon}} X_{3}
\end{aligned}
$$

where $X_{2}$ catalyses the fast forward conversion of $Y_{1}$ into $X_{3}$. The position of $X_{2}$ and $X_{3}$ could also be exchanged to give a third possibility

$$
\begin{aligned}
& \emptyset \stackrel{\frac{K}{\epsilon}}{\longrightarrow} Y_{1} \stackrel{\frac{\delta}{\epsilon}}{\longrightarrow} \emptyset \\
& Y_{1} \underset{\frac{\alpha x_{3}}{\epsilon}}{\stackrel{\frac{\beta}{\epsilon}}{\epsilon}} X_{2} .
\end{aligned}
$$


If the parameters of the latter two fast layers are tuned such that $\alpha K / \beta \delta=1$, the conservation relation $x_{2}=x_{3}$ results from any of these implementations.

However, the column spaces $\operatorname{Col}\left(S^{f}\right)$ of these implementations imply vastly different behavior. In (14a) both $X_{2}$ and $X_{3}$ have dynamics with fast and slow components. Hence, the coupling between the two modules implemented by this fast layer is bidirectional; we conserve $x_{2}=x_{3}$ such that the slow dynamics of either species will drive the slow dynamics of the other. Tuning the parameters used in $R_{1}(x)$ and $R_{2}(x)$ will therefore affect the behavior of $X_{3}$ and $X_{4}$, and vice versa.

However, in the fast layer (14b) there is no $x_{2}$ component in $\operatorname{Col}\left(S^{f}\right)$. Therefore the dynamics of $x_{2}$ in the approximated system equal the isolated slow dynamics of $x_{2}$. Thus, the $X_{3} / X_{4}$ module is downstream of the $X_{1} / X_{2}$ module, such that the dynamics of $x_{3}$ no longer depend on $R_{3}(x), \alpha_{3}$ or $\delta_{3}$. Instead, $x_{3}$ is driven by $x_{2}$. Similarly, (14c) implements the reverse cascade structure, so that $x_{2}$ is driven by $x_{3}$. Hence, the appropriate design of the fast layer can propagate signals between modules in either direction, or both.

Importantly, none of these implementations require the interaction terms $R_{i}$ to be remodelled. Furthermore, nonlinear conservations (such as those discussed in Sections IV-A and IV-B) may couple modules in more interesting ways than $x_{2}=x_{3}$. Additionally, modules may be connected through a combination of actuation (i.e., GRN design) and through coupling (i.e., PIN coupling of modules). The details of this combined design strategy will form the basis of further work on this approach.

\section{DISCUSSION}

The goal of this paper has been the design of fast layers that enforce specified conservation relations on a biosynthetic network's state values. It is natural to consider to what extent a general constraint $p(x(t)) \equiv 0$ can be satisfied by this approach. Section III shows that the $r^{f}$ independent nonlinear conservation relations $\lambda_{M} \in \operatorname{Row}\left(M^{f}(x)\right)$ are equivalent to $C^{f} v^{f}(x(t)) \equiv 0$. Thus, the fast layer may be designed such that a linear combination of these conservation relations matches the required constraint. However, if this is not possible, the implementation of $x_{3}=\gamma x_{1}^{2}$ by (13) demonstrates how constraints can also be implemented as the intersection of multiple independent conservation relations (see also Section V-A-1).

It is beyond the scope of this paper to formulate all possible implementations of general $p(x(t)) \equiv 0$ as a fast layer. Section IV demonstrates that there are can be multiple appropriate implementations for a given $p$. Although the constraints considered above are polynomial, other non-polynomial conservation relations could conceivably be implemented by fast reaction rates not modelled by mass-action kinetics. Thus, this work is a first step towards a systematic design strategy for arbitrary conservation relations.

\section{A. Fast Layer Structure}

The state values of a given network can be constrained by imposing $r^{f}$ tunable conservation relations through a fast layer. While the flux vector $v^{f}$ of the fast layer determines the shape of these conservation relations, we have seen in Section IV that the stoichiometric matrix of the fast layer is also important in a number of ways.

1) Fast Intermediates: Recall that the latter two implementations of inter-modular coupling in (14) used an additional intermediate species $Y_{1}$. This can be a useful way of combining together multiple conservation relations. For example, suppose we wish to implement $K=\alpha x_{1} x_{2}$. By introducing a new intermediate species $Y_{1}$ into the system, this conservation relation can be implemented by

$$
\begin{aligned}
& \emptyset \stackrel{\frac{\kappa_{1}}{\epsilon}}{\longrightarrow} Y_{1} \stackrel{\frac{\kappa_{2}}{\epsilon}}{\longrightarrow} \emptyset \\
& X_{1}+X_{2} \underset{\frac{\frac{\beta}{\epsilon}}{\rightleftharpoons}}{\stackrel{\frac{\alpha}{\epsilon}}{\rightleftharpoons}} Y_{1} .
\end{aligned}
$$

The two conservation relations $y_{1}=\kappa_{1} / \kappa_{2}$ and $\alpha x_{1} x_{2}=\beta y_{1}$ can be combined to give the required conservation $K=\alpha x_{1} x_{2}$, where the constants $K=\beta \kappa_{1} / \kappa_{2}$ and $\alpha$ depend on the fast layer's parameters.

2) Steady State Fluxes: The existence of multiple implementations of the same conservation relation has been observed in each of the preceding examples. The choice of implementation has an important effect on the system's dynamics under the specified constraint.

For a general reaction network (1) the right null space $\operatorname{ker}(S)$ defines the space of fluxes $v \in \mathbb{R}^{m}$ for which the system is at steady state. If the network can be written in the layered form (2), we can approximate this system by (5) and instead consider the rates $v^{s} \in \mathbb{R}^{m^{s}}$ of the $m^{s}$ slow reactions as the system's flux vector.

The space of steady state fluxes $v^{s}$ for (5) is

$$
\mathcal{V}=\left\{v^{s} \in \mathbb{R}^{m^{s}} \mid S^{s} v^{s} \in \operatorname{ker}\left(P^{f}(x)\right)=\operatorname{Col}\left(S^{f}(x)\right)\right\}
$$

which is a vector subspace of $\mathbb{R}^{m^{s}}$ that includes $\operatorname{ker}\left(S^{s}\right)$. If $\operatorname{Col}\left(S^{f}\right) \cap \operatorname{Col}\left(S^{s}\right)=\{0\}$ then $\mathcal{V}=\operatorname{ker}\left(S^{s}\right)$. However, if $\operatorname{Col}\left(S^{f}\right) \cap \operatorname{Col}\left(S^{s}\right)$ has positive dimension, then the dimension of $\mathcal{V}$ will be larger than the dimension $m^{s}-r^{s}$ of $\operatorname{ker} S^{s}$. Incorporating a fast layer thus increases the space of steady-state flux distributions.

The space $\mathcal{V}$ identifies the slow fluxes that are 'projected out' by the fast layer. Consider extending the state of the GRN example with extra species $Y_{1}$, so that the slow layer's stoichiometric matrix is $S^{s}=\left[I_{n}, \underline{0}\right]^{T}$ for the $n \times n$ identity $I_{n}$ and zero vector $\underline{0} \in \mathbb{R}^{n}$. For the fast layer (14b)

$$
\mathcal{V}=\operatorname{Span}\left(\left[\begin{array}{llllll}
0 & 0 & 1 & 0 & \ldots & 0
\end{array}\right]^{T}\right)
$$

Therefore $v^{s}=\left(0,0, v_{3}, \ldots, 0\right)^{T} \in \mathcal{V}$ for any slow reaction $v_{3}$ given by (9). Hence the $v_{3}$ component of any slow flux vector $v^{s}$ is sent to zero by (5), and the resulting dynamics are independent of $\alpha_{3}, \delta_{3}$, and $R_{3}(x)$.

The set $\mathcal{V}$ implies that the fast layer's structure can expand the space of steady state slow fluxes for the layered system, and hence will influence the design of the slow layer. This dual problem will be an important topic of further research in layered approaches to Synthetic Biology.

3) Replacing Existing Conservation Relations: In isolation, the GRN (9) used as the example slow layer has no conservation relations, as $\operatorname{LNS}\left(S^{s}\right)=\{0\}$. Hence the conservation relations 
of the combined system are defined by $\operatorname{Row}\left(M^{f}(x)\right)$. Implementing a fast layer of dimension $r^{f}$ thus results in $r^{f}$ nonlinear conservation relations. However, other slow layers may have positive dimension $n-r^{s}$ of $\operatorname{LNS}\left(S^{s}\right)$, so that further conservation relations may then be contributed by $\operatorname{LNS}\left(S^{f}\right) \cap \operatorname{LNS}\left(S^{s}\right)$. The fast layer's stoichiometric structure therefore becomes important for selecting which of the slow layer's original $n-r^{s}$ conservation relations remain valid for the approximation (5).

Relaxing the assumption that $r^{s}=n$ therefore extends the impact of the fast layer from constraining a given state space (with extra conservation relations) to also removing existing conservation relations. Further work is necessary to determine how best to exploit this additional flexibility.

\section{B. Slow Layer Design}

We have shown how conservation relations can be implemented independently of the slow layer's parameter values. Although there has been a great deal of work on parameter tuning in Synthetic Biology, it is not yet clear how the design of state space constraints will combine with that of slow dynamics. Once a conservation relation is implemented, how should the slow system be tuned? Indeed, is there a conservation relation that would make the design of a specific behavior in a given slow network any easier?

Furthermore, slow reactions are often easier to tune than a fast layer. In this case, a slow layer may be constructed to achieve a design specification given a fixed fast layer. This strategy underlies the genetic control of metabolic networks, where a synthetic genetic network steers the steady state of a metabolic network to a desired configuration [25], [26]. A key goal of future work is to investigate strategies for the combined design of fast and slow layers to produce specific, complex behaviors.

\section{Experimental Implementation}

As an example of networks with layers that separate in timescale, we have considered a GRN layer combined with a faster PIN layer. Designing the resulting conservation relations requires control of the topology and reaction rates of the protein interactions. Recent studies have identified various means by which transcription factors and related proteins and microRNAs interact at the post-translational level [41]-[43].

A number of methods can modulate these protein interactions [3], for example using small molecules [44] and protein engineering techniques [11], [12] to interrupt dimerization affinities. Other fast post-transcriptional interactions such as phosphorylation can be modulated by kinase and phosphotase levels. However, any modifications to TFs may cause unexpected crosstalk within the PIN layer, or also affect their regulatory behavior. Such context-dependent failure [13] is caused by the non-modularity of protein design, which will provide an important challenge for the experimental implementation of a layered design strategy.

\section{Stochasticity}

A vital issue for experimental implementation is the effect of noise, which our framework has so far neglected. Due to stochasticity, the fast layer's nonlinear conservation relations hold only in expectation; the fast layer's contribution $x^{f}\left(x^{s}\right)$ quickly approaches a steady-state distribution that varies slowly with $x^{s}$. It has been shown in [29] that, for the linear noise approximation, the statistics of the fluctuations of fast variables around a slow manifold do not match the heuristic derivation of a stochastic version of the reduced deterministic model, and should instead be calculated by reducing the full stochastic model. More recently, conditions quantifying the accuracy of the heuristic derivation were identified [30].

An important development for the further investigation of layered design is the probabilistic interpretation of tuning the fast reactions such that the state values are distributed in a region of state space around the deterministic slow manifold. Similarly to shaping this manifold, it may also be possible to shape the distribution by the design of the fast layer. We could use a heuristic stochastic model based on the approximate system (5), but the results of [29], [30] suggest instead that we should extend the decomposition described in [21] to reduce layered stochastic models.

\section{CONCLUSION}

In a biomolecular network, layered by timescale, the fast layer constrains the state space through conservation relations valid beyond a fast transient. In contrast to systems on one timescale, a number of these conservation relations are, in general, parameter-dependent and nonlinear. Hence, conservation relations can be designed by constructing an appropriately structured and tuned fast layer. This principle can be used to independently design additional fast reactions that constrain a slow system's state values to specification.

\section{REFERENCES}

[1] A. L. Slusarczyk, A. Lin, and R. Weiss, "Foundations for the design and implementation of synthetic genetic circuits," Nature Rev. Genetics, vol. 13, pp. 406-420, 2012.

[2] P. E. Purnick and R. Weiss, "The second wave of synthetic biology: From modules to systems," Nature Rev. Mol. Cell Biol., vol. 10, pp. 410-422, 2009.

[3] J. A. J. Arpino, E. J. Hancock, J. Anderson, M. Barahona, G.-B. V. Stan, A. Papachristodoulou, and K. Polizzi, "Tuning the dials of synthetic biology," Microbiology, vol. 159, pp. 1236-1253, 2013.

[4] M. R. Atkinson, M. A. Savageau, J. T. Myers, and A. J. Ninfa, "Development of genetic cicircuit exhibiting toggle switch or oscillatory behavior in Escherichia coli," Cell, vol. 113, no. 5, pp. 597-607, 2003.

[5] R. Daniel, J. R. Rubens, R. Sarpeshkar, and T. K. Lu, "Synthetic analog computation in living cells," Nature, 2013.

[6] T. Ellis, X. Wang, and J. J. Collins, "Diversity-based, model-guided construction of synthetic gene networks with predicted functions," $\mathrm{Na}$ ture Biotechnol., vol. 27, pp. 465-471, 2009.

[7] H. N. Lim, Y. Lee, and R. Hussein, "Fundamental relationship between operon organization and gene expression," Proc. Nat. Acad. Sci., vol. 108, no. 26, pp. $10626-10631,2011$.

[8] E. Yeung, A. Ng, J. Kim, Z. Z. Sun, and R. M. Murray, "Modeling the effects of compositional context on promoter activity in an E. coli extract based transcription-translation system," in Proc. IEEE Conf. Decision and Control, 2014, pp. 5405-5412.

[9] R. G. Egbert and E. Klavins, "Fine-tuning gene networks using simple sequence repeats," Proc. Nat. Acad. Sci., vol. 109, no. 42, pp. 16 817-16 822, 2012.

[10] H. M. Salis, E. A. Mirsky, and C. A. Voigt, "Automated design of synthetic ribosome binding sites to control gene expression," Nature Biotechnol., vol. 27, no. 10, pp. 946-950, 2009.

[11] J. A. Brannigan and A. J. Wilkinson, "Protein engineering 20 years on," Nature Rev. Mol. Cell Biol., vol. 3, pp. 964-970, 2002.

[12] M. Heinemann and S. Panke, "Synthetic biology-Putting engineering into biology," Bioinformatics, vol. 22, no. 22, pp. 2790-2799, 2006. 
[13] S. Cardinale and A. P. Arkin, "Contextualizing context for synthetic biology-Identifying causes of failure of synthetic biological systems," Biotechnol. J., vol. 7, pp. 856-866, 2012.

[14] N. Jamshidi and B. O. Palsson, "Top-down analysis of temporal hierarchy in biochemical reaction networks," PLoS Comput. Biol., vol. 4, no. 9, p. e1000177, 2008.

[15] M. R. Bennett, D. Volfson, L. Tsimring, and J. Hasty, "Transient dynamics of genetic regulatory networks," Biophys. J., vol. 92, pp. 3501-3512, 2007.

[16] A. Ciliberto, F. Capuani, and J. J. Tyson, "Modeling networks of coupled enzymatic reactions using the total quasi-steady state approximation," PLoS Comput. Biol., vol. 3, no. 3, p. e45, 2007.

[17] C. H. Lee and H. G. Othmer, "A multi-time-scale analysis of chemical reaction networks: Deterministic systems," J. Math. Biol., vol. 60, no. 3, pp. 387-450, 2010.

[18] N. Vora and P. Daoutidis, "Nonlinear model reduction of chemical reaction systems," AIChE J., vol. 47, no. 10, pp. 2320-2332, 2001.

[19] N. P. Vora, M.-N. Contou-Carrere, and P. Daoutidis, "Model reduction of multiple time scale processes in non-standard singularly perturbed form," in Model Reduction and Coarse-Graining Approaches for Multiscale Phenomena, A. N. Gorban, N. K. Kazantzis, I. G. Kevrekidis, H. C. Öttinger, and C. Theodoropoulos, Eds. New York, NY, USA: Springer, 2006.

[20] Z. P. Gerdtzen, P. Daoutidis, and W.-S. Hu, "Non-linear reduction for kinetic models of metabolic reaction networks," Metab. Eng., vol. 6, pp. 140-154, 2004.

[21] T. P. Prescott and A. Papachristodoulou, "Layered decomposition for the model order reduction of timescale separated biochemical reaction networks," J. Theor. Biol., vol. 356, pp. 113-122, 2014.

[22] D. Del Vecchio, A. J. Ninfa, and E. D. Sontag, "Modular cell biology: Retroactivity and insulation," Mol. Syst. Biol., vol. 4, p. 161, 2008.

[23] S. Jayanthi and D. Del Vecchio, "Retroactivity attentuation in biomolecular systems based on timescale separation," IEEE Trans. Autom. Control, vol. 56, no. 4, pp. 748-761, 2011.

[24] P. M. Rivera-Ortiz and D. Del Vecchio, "Integral action with time scale separation: A mechanism for modularity in biological systems," in Proc. IEEE Conf. Decision and Control, 2014, pp. 49-55.

[25] J. Kuntz, D. A. Oyarzún, and G.-B. Stan, "Model reduction of genetic-metabolic networks via time scale separation," in A Systems Theoretic Approach to Systems and Synthetic Biology I: Models and System Characterizations, V. V. Kulkarni, G.-B. Stan, and K. Raman, Eds. Dordrecht, Germany: Springer, 2014.

[26] S. Waldherr, D. A. Oyarzún, and A. Bockmayr, "Dynamic optimization of metabolic networks coupled with gene expression," J. Theor. Biol., vol. 365, pp. 469-485, 2015.

[27] T. P. Prescott and A. Papachristodoulou, "Layering in networks: The case of biochemical systems," in Proc. Amer. Control Conf., 2013, pp. 4544-4549.

[28] T. P. Prescott and A. Papachristodoulou, "Signal propagation across layered biochemical networks," in Proc. Amer. Control Conf., 2014, pp. 3399-3404.

[29] P. Thomas, A. V. Straube, and R. Grima, "The slow-scale linear noise approximation: An accurate, reduced stochastic description of biochemical networks under timescale separation conditions," $B M C$ Syst. Biol., vol. 6, p. 39, 2012.

[30] J. K. Kim, K. Josić, and M. R. Bennett, "The validity of quasi-steadystate approximations in discrete stochastic simulations," Biophys. J., vol. 107, pp. 783-793, 2014.

[31] B. O. Palsson, Systems Biology: Properties of Reconstructed Networks. Cambridge, U.K.: Cambridge Univ. Press, 2006.

[32] M. Feinberg and F. J. M. Horn, "Dynamics of open chemical systems and the algebraic structure of the underlying reaction network," Chem. Eng. Sci., vol. 29, no. 3, pp. 775-787, 1974.

[33] J. Hasty, F. J. Isaacs, M. Dolnik, D. McMillen, and J. J. Collins, "Designer gene networks: Towards fundamental cellular control," Chaos, vol. 11 , no. 1 , pp. $207-220,2001$.

[34] D. J. M. Park, "The hierarchical structure of metabolic networks and the construction of efficient metabolic simulators," J. Theor. Biol., vol. 46, pp. 31-74, 1974.
[35] R. Heinrich and S. Schuster, The Regulation of Cellular Systems. London, U.K.: Chapman \& Hall, 1996.

[36] P. V. Kokotovic, J. J. Allemong, J. R. Winkelman, and J. H. Chow, "Singular perturbation and iterative separation of time scales," Automatica, vol. 16, pp. 23-33, 1980.

[37] T. J. Kaper, "An introduction to geometric methods and dynamical systems theory for singular perturbation problems," in Proc. Symp. Applied Mathematics, 1999, vol. 56, pp. 85-132, AMS.

[38] R. Marino and P. V. Kokotovic, "A geometric approach to nonlinear singularly perturbed control systems," Automatica, vol. 24, no. 1, pp. 31-41, 1988.

[39] E. Yeger-Lotem, S. Sattath, N. Kashtan, S. Itzkovitz, R. Milo, R. Y. Pinter, U. Alon, and H. Margalit, "Network motifs in integrated cellular networks of transcription-regulation and protein-protein interaction," Proc. Nat. Acad. Sci., vol. 101, no. 16, pp. 5934-5939, 2004.

[40] A. Gyorgy and D. Del Vecchio, "Modular composition of gene transcription networks," PLoS Comput. Biol., vol. 10, p. e1003486, 2014.

[41] C. A. Grove and A. J. M. Walhout, "Transcription factor functionality and transcription regulatory networks," Mol. BioSyst., vol. 4, pp. 309-314, 2008.

[42] N. J. Martinez and A. J. M. Walhout, "The interplay between transcription factors and microRNAs in genome-scale regulatory networks," BioEssays, vol. 31, pp. 435-445, 2009.

[43] D. Y. Rhee, D.-Y. Cho, B. Zhai, M. Slattery, L. Ma, J. Mintseris, C. Y. Wong, K. P. White, S. E. Celniker, T. M. Przytycka, S. P. Gygi, R. A. Obar, and S. Artavanis-Tsakonas, "Transcription factor networks in Drosophila melanogaster," Cell Reports, vol. 8, pp. 2031-2043, 2014.

[44] G. D. Amoutzias, D. L. Robertson, Y. V. de Peer, and S. G. Oliver, "Choose your partners: Dimerization in eukaryotic transcription factors," Trends Biochem. Sci., vol. 33, no. 5, pp. 220-229, 2008.

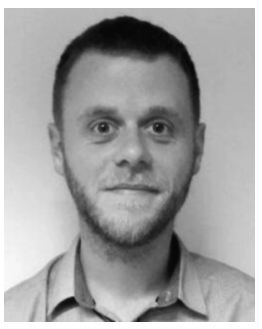

Thomas P. Prescott received the MMath degree in mathematics and the D.Phil. degree in engineering science from the University of Oxford, Oxford, U.K., in 2008 and 2015, respectively.

Currently, he is a Postdoctoral Research Assistant in Control Engineering in the Department of Engineering Science at the University of Oxford. His research interests include mathematical modelling of evolved and synthetic biological systems, and other large-scale systems of nonlinear differential equations.

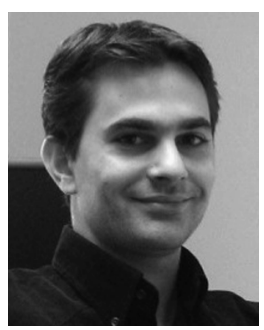

Antonis Papachristodoulou received the M.A./M.Eng. degree in electrical and information sciences from the University of Cambridge, Cambridge, U.K., and the Ph.D. degree in control and dynamical systems (with a minor in aeronautics) from the California Institute of Technology, Pasadena, CA, USA.

Currently, he is an Associate Professor in Engineering Science at the University of Oxford, Oxford, U.K., and a Tutorial Fellow at Worcester College, Oxford, U.K. He is an EPSRC Fellow for Growth in Synthetic Biology and Director of the EPSRC \& BBSRC Centre for Doctoral Training in Synthetic Biology. His research interests include large-scale nonlinear systems analysis, sum of squares programming, synthetic and systems biology, networked systems, and flow control.

Prof. Papachristodoulou received the 2015 European Control Award for his contributions to robustness analysis and applications to networked control systems and systems biology. In the same year, he received the O. Hugo Schuck Best Paper Award. 